\title{
ECG Biometric Identification Method based on Parallel 2-D Convolutional Neural Networks
}

\author{
Ayça Hanilçi iD, Hakan Gürkan* iD
}

Bursa Technical University, Department of Electrical and Electronics Engineering, 16310 Bursa, Turkey

\author{
Cite this paper as: \\ Hanilci, A., Gürkan, H., (2019). ECG \\ Biometric Identification Method based on \\ Parallel 2-D Convolutional Neural \\ Networks. Journal of Innovative Science \\ and Engineering. 3(1): 11-22 \\ *Corresponding author: Hakan Gürkan \\ E-mail: hakan.gurkan@btu.edu.tr \\ Tel: +90 (224) 3003797 \\ Received Date: 30/04/2019 \\ Accepted Date: 29/05/2019 \\ (C) Copyright 2019 by \\ Bursa Technical University. Available \\ online at http://jise.btu.edu.tr/

\section{(c) (i) (5)} \\ The works published in Journal of \\ Innovative Science and Engineering \\ (JISE) are licensed under a Creative \\ Commons Attribution-NonCommercial 4.0 \\ International License.
}

\begin{abstract}
In this paper, an ECG biometric identification method, based on a two-dimensional convolutional neural network, is introduced for biometric applications. The proposed model includes two-dimensional convolutional neural networks that work parallel and receive two different sets of 2-dimensional features as input. First, ACDCT features and cepstral properties are extracted from overlapping ECG signals. Then, these features are transformed from one-dimensional representation to two-dimensional representation by matrix manipulations. For feature learning purposes, these two-dimensional features are given to the inputs of the proposed model, separately. Finally, score level fusion is applied to identify the user. Our experimental results show that the proposed biometric identification method achieves an accuracy of $\% 88.57$ and an identification rate of $90.48 \%$ for 42 persons.
\end{abstract}

Keywords: Biometric identification; Electrocardiogram Signal; CNN 


\section{Introduction}

The electrocardiogram (ECG) is a low-cost biological signal that can be measured on the skin and indicates the electrical activity of the heart [1-3]. Over the last thirty years, the analysis of ECG signals have become a very important tool for clinical diagnosis and has been an important research area [2].

Biometric recognition is a task of identifying an individual using his/her physiological and/or behavioral characteristics [1]. Physiological features are usually constant and do not change easily over time. However, features based on behavioral characteristics may vary depending on time and environment. Over the last thirty years, biometric recognition systems have been developed based on physiological characteristics such as face, fingerprint, hand geometry, iris, and retina or behavioral characteristics such as walking pattern, signature, and speech $[2,3]$.

Recent studies show that ECG signal, which is a very important tool for clinical diagnosis, can be used as a new biometric modality. ECG signals have distinctive characteristics, especially due to various personal factors such as age, gender, position, size and anatomy of the heart. For this reason, ECG signals have been used in biometric recognition systems $[2,4]$. The most important advantage of ECG-based biometric recognition systems in terms of reliability is that these signals must be obtained from a living body [3]. Besides, the ECG, which is a universal signal, is a sign of life that is almost impossible to imitate and shows that the person is physically present there. In addition, a technology that can mimic or produce an ECG signal of a person has not been developed yet. Therefore, ECG based biometric recognition systems have a potential to be used in the areas such as computer and network access controls, cash machine systems, and border control systems [2].

Over the past two decades, various ECG based biometric recognition systems and applications have been proposed [230]. In addition to these conventional methods, as results of the increase in computer operating speeds and the development of graphical processor units (GPUs), deep learning-based methods have been widely used in biometric recognition for feature extraction and classification [31]. Especially in recent years, convolutional neural network (CNN) models which is one of the deep learning methods for machine learning has been used for ECG based biometric recognition. [32-39].

In 2016, Lei et.al. [32] proposed a one-dimensional CNN model for feature extraction and a nonlinear support vector machine (SVM) model for classification. The proposed method achieved an accuracy of $99.33 \%$ using a database consisting of 100 people [32]. In 2017, Zhang et.al. [33] introduced a one-dimensional parallel CNN model for feature extraction and classification. The model has been tested on different ECG datasets and performed an average recognition rate of 93.5\% [33]. Eduardo et.al. [34] presented a feature extraction method based on deep autoencoder and K-Nearest Neighborhood (K-NN) method for classification. The proposed model was tested on a database of 790 people and $0.91 \%$ identification error was reported [34]. Salloum et.al. developed a recurrent neural network (RNN) based model for feature extraction and classification. Their experimental results showed that the model performed $100 \%$ accuracy for two different ECG datasets consisting of 47 and 90 people [35]. In the same year, a two dimensional CNN model using ECG trajectory images of 200x200 pixels was proposed by Zhang et.al. [36]. The proposed CNN model was tested on 
a 10-person ECG dataset and a 98.4\% recognition rate was obtained. [36]. In 2018, Luz et.al. [37] proposed a model in which one-dimensional and two-dimensional CNN structures were used together. In this model, QRS segments aligned according to R points were applied as input to one dimensional CNN structure. At the same time, the spectrograms of these QRS segments were applied to the input of the two-dimensional CNN structure. The proposed model performed an equal error rate of 14.3\% [37]. In 2018 Labati et.al. [38] developed ECG biometric recognition method based on onedimensional CNN structure. The model was tested on a data set of 52 people and achieved a $100 \%$ identification rate [38]. In the same year, Abdeldayem et.al. [39] presented a two-dimensional CNN model which is fed with the spectrotemporal images extracted by using short-term Fourier transform (STFT) and continuous wavelet transform (CWT). The proposed model achieved an accuracy of $97.5 \%$ [39].

In this paper, an ECG biometric identification method based on a two-dimensional convolutional neural network (2D$\mathrm{CNN}$ ) is proposed. The proposed model consists of two parallel 2D-CNN's. In the proposed model, ACDCT features and cepstral properties are first extracted from overlapping ECG signals. These features are then transformed from 1D to $2 \mathrm{D}$ by matrix manipulations. For feature learning purposes, these $2 \mathrm{D}$ features are given to the inputs of two $2 \mathrm{D}$ CNN's, separately. Finally, score level fusion is applied to identify the user.

\section{Material and Methods}

The proposed method consists of four main parts: data acquisition, signal preprocessing, feature extraction, and classification.

\subsection{ECG Database}

The Physikalisch-Technische Bundesanstalt (PTB) Diagnostic ECG Database [40, 41] was selected for constructing training and testing datasets. The main reason for selecting this database is that ECG records for the same person are collected on different days [2, 4]. The database consists of 549 ECG records collected from 290 people who have one to five ECG records which sampled at $1 \mathrm{kHz}$ with a resolution of 16-bit. Each record in the database includes the twelve conventional leads and three Frank leads [2]. In the current experiment, Lead-I that is recorded between two wrists was used because the configuration is more suitable for biometric applications [20].

In this experiment, 42 people, who have at least four ECG records from the database, were selected. Then, training dataset was constructed using three of the ECG records for each selected person while test dataset was built using the other record.

\subsection{Signal Preprocessing}

In order to improve the classification performance, ECG signals should be prepared by filtering and segmentation steps before the feature extraction steps. This process is known as signal preprocessing.

In the first step, ECG signals which are affected by noise such as baseline wander, power noise, and high-frequency noise are filtered utilizing a band-pass filter with cut-off frequencies at $1 \mathrm{~Hz}$ and $40 \mathrm{~Hz}$. In the second step, the output of the band-pass filter is divided into 50\% overlapping frames to increase the correlation between consecutive frames. For an ECG signal sampled at $1 \mathrm{kHz}$, each frame with 5000 samples can be described as follows: 


$$
x_{\text {normseg }}=\left[\begin{array}{c}
x_{\text {normseg }}(1) \\
x_{\text {normseg }}(2) \\
\vdots \\
x_{\text {normseg }}(5000)
\end{array}\right]=\left[\begin{array}{c}
x_{\text {normseg }}((i-1) \times 2500+1) \\
x_{\text {normseg }}((i-1) \times 2500+2) \\
\vdots \\
x_{\text {normseg }}((i+1) \times 2500)
\end{array}\right], i=1,2, \ldots, N_{F}
$$

\subsection{Feature Extraction}

The proposed system processes two different feature sets as inputs. The first one is ACDCT features which were proposed by Wang et al. [2]. The ACDCT coefficients are calculated by applying the autocorrelation followed by discrete cosine transform (DCT) to the ECG signal. After the autocorrelation process, obtained coefficients are normalized with respect to the first lag to remove biasing effect as follows:

$$
\begin{gathered}
\hat{r}(m)=\sum_{n=0}^{L_{F}-|m|-1} x_{\text {normseg }}(n) x_{\text {normseg }}(n+m) \quad, \quad m=0,1,2, \ldots, L-1 \\
r(m)=\frac{\hat{r}(m)}{\hat{r}(0)}
\end{gathered}
$$

where $\boldsymbol{r}(\boldsymbol{m}), \boldsymbol{L}$, and $\boldsymbol{L}_{\boldsymbol{F}}$ represent the autocorrelation coefficients, the length of the sequence of the autocorrelation coefficients, and the length of the frame of the ECG signal, respectively. Afterwards, the DCT is applied to autocorrelation coefficients as follows:

$$
\begin{gathered}
c(u)=\alpha(u) \sum_{m=0}^{L-1} r(m) \cos \left[\frac{\pi u}{L}\left(m+\frac{1}{2}\right)\right] \\
\alpha(u)= \begin{cases}\sqrt{1 / L} & \text { for } u=0 \\
\sqrt{2 / L} & \text { for } u \neq 0\end{cases}
\end{gathered}
$$

where $\boldsymbol{c}(\boldsymbol{u})$ represents the ACDCT feature set [2].

In addition to ACDCT features, we used cepstral features as the second feature set to represent the frequency characteristics of the ECG signals. For this purpose, Mel-Frequency Cepstral Coefficients (MFCC) extraction method, which is a widely used feature extraction method in speech processing [42-44], was selected and adapted to ECG signals. The Discrete Fourier Transform (DFT) coefficients are first calculated for each ECG frame as follows: 


$$
X(k)=\sum_{n=0}^{N-1} x_{n o r m s e g}(n) e^{-j 2 \pi n k / N}, k=0,1,2, \ldots, N-1
$$

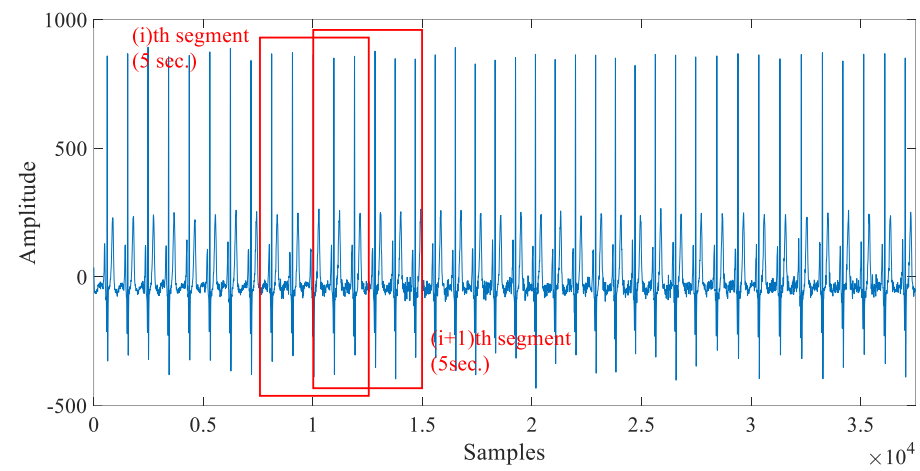

Figure 1. ECG segments

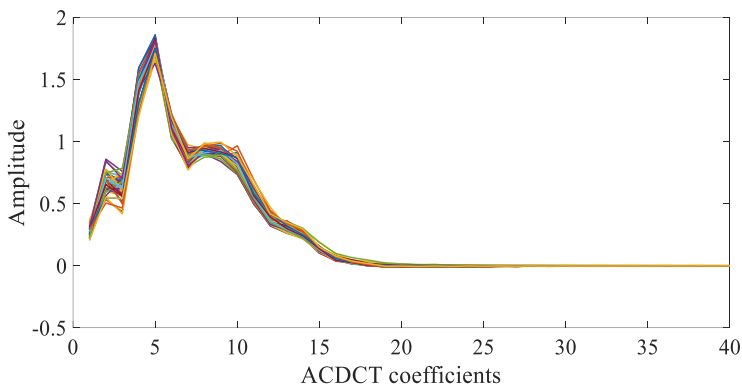

Figure 2. ACDCT feature vectors

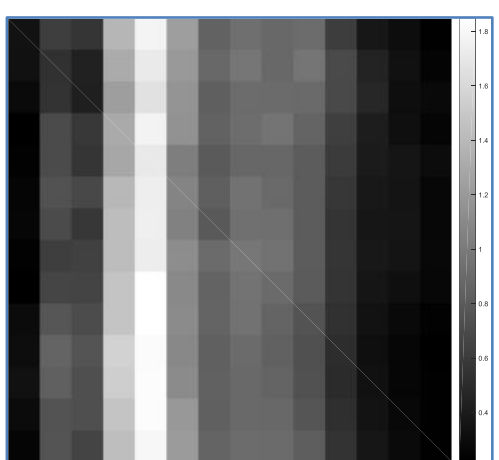

Figure 4. ACDCT feature image

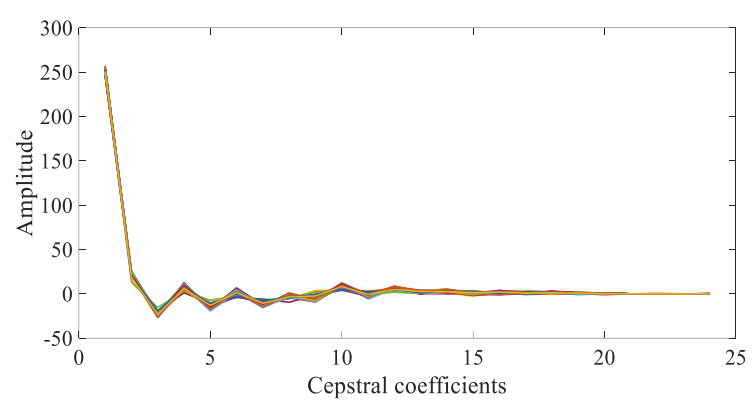

Figure 3. Cepstral feature vectors

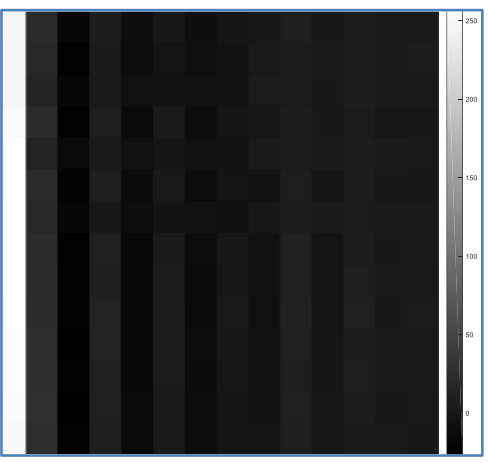

Figure 5. Cepstral feature image

The DFT coefficients are then applied to the input of the filterbank that consists of M-triangle filter [42-44]. At this point, it should be emphasized that the filters in the filter bank are uniformly placed in the linear frequency band between 
$1 \mathrm{~Hz}$ and $40 \mathrm{~Hz}$ due to the frequency characteristics of the ECG signals, unlike the application in speech processing. After that, the cepstral features are computed by applying DCT to the output of the filterbank [42-44] as follows:

$$
\begin{gathered}
S(m)=\ln \left[\sum_{k=0}^{N-1}|X(k)| H_{m}(k)\right], m=0,1,2, \ldots, M-1 \\
C C(l)=\sum_{m=0}^{M-1} S(m) \cos \left[\frac{\pi l}{M}\left(m+\frac{1}{2}\right)\right], \quad l=0,1,2, \ldots, M-1
\end{gathered}
$$

where, $X(k), H_{m}(k), S(m)$, and $C C(l)$ represent the magnitude spectrum of each frame, the filterbank with M-triangle filters, the output of the filterbank, and the cepstral coefficients, respectively.

Finally, $M$ one-dimensional feature vectors with $M$ coefficients were obtained for $M$ overlapped ECG segments by the process described above. Then, these feature vectors were transformed to two-dimensional $(M \times M)$ feature images by matrix manipulation as shown in Figure 1-5.

\subsection{Classification using CNN}

The proposed CNN architecture is composed of two 2D-CNN connected in parallel shown in Figure 6. Each 2D-CNN consists of two convolutional layers with rectified linear unit (ReLU), one max-pooling layer, two dropout layers, a fully connected layer, and a softmax layer. The detail of the proposed CNN architecture is given in Table 1.

Table 1. CNN architecture used in the ECG biometric identification system

\begin{tabular}{cccccc}
\hline & Layer Type & $\begin{array}{c}\text { Activation } \\
\text { Unit }\end{array}$ & $\begin{array}{c}\text { Kernel } \\
\text { Size }\end{array}$ & Output Size & $\begin{array}{c}\text { Number of } \\
\text { Parameters }\end{array}$ \\
\hline $\mathbf{1}$ & Convolutional & ReLU & $3 \times 3$ & $14 \times 14,32$ & 320 \\
\hline $\mathbf{2}$ & Convolutional & ReLU & $3 \times 3$ & $14 \times 14,64$ & 18496 \\
\hline $\mathbf{3}$ & Max. Pooling & - & $2 \times 2$ & $7 \times 7,64$ & - \\
\hline $\mathbf{4}$ & Dropout $(0.25)$ & - & - & $7 \times 7,64$ & - \\
\hline $\mathbf{5}$ & Flaten & - & - & $1 \times 3136$ & - \\
\hline $\mathbf{6}$ & Fully-connected & ReLU & - & $1 \times 128$ & 401536 \\
\hline $\mathbf{7}$ & Dropout $(0.5)$ & - & - & $1 \times 128$ & - \\
\hline $\mathbf{8}$ & Output & Softmax & - & 42 & 5418 \\
\hline
\end{tabular}

As illustrated in Figure 6, each 2D-CNN is fed with $14 \times 14$ images which are generated as the output of the preprocessing and feature extraction steps. After obtaining the scores at the output of each 2D-CNN, the mean-value based score fusion technique is applied to identify the user. 


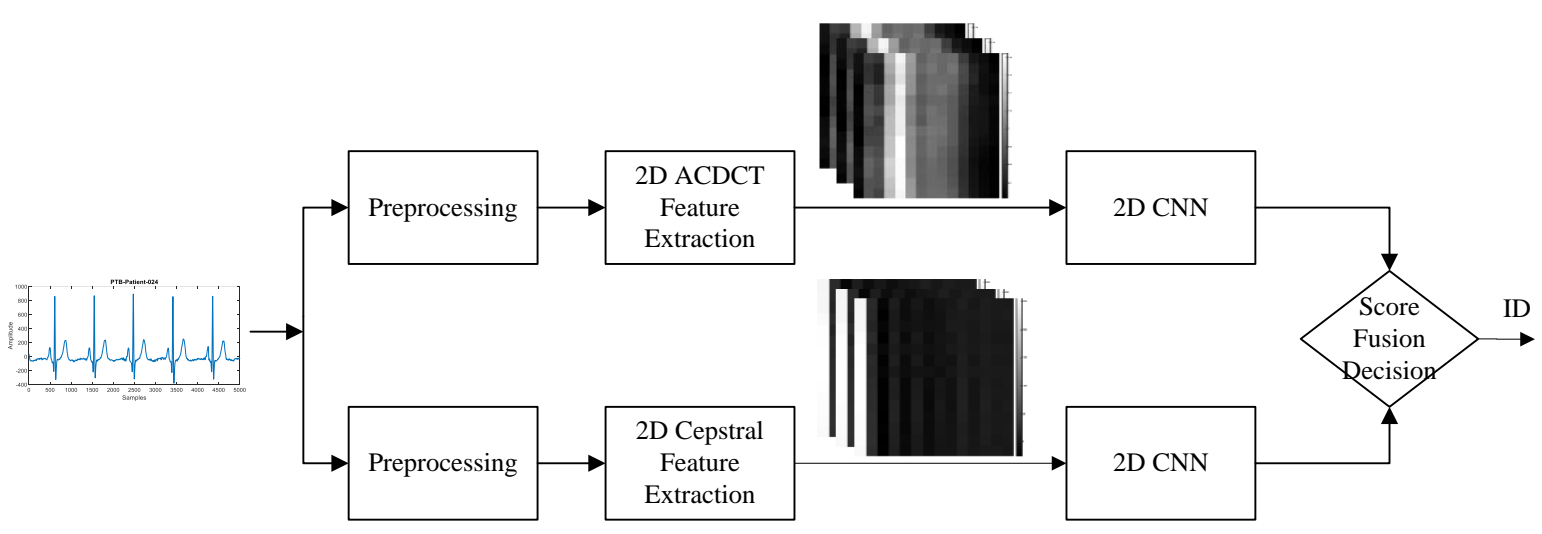

Figure 6. Proposed CNN based ECG biometric identification method

\section{Experimental Results and Discussion}

In our experiment, 168 ECG signals for 42 different people are used, each with four ECG signals. After preprocessing and feature extraction process, twenty ACDCT images and twenty CC images with a dimension of $14 \times 14$ for each person are used. First, each 2D-CNN model, without data augmentation, using 630 images (42x15) is trained. Then, each model using 210 images (42x5) is tested in the same condition. Afterward, the performance of the proposed method is evaluated by using five statistical measures: identification rate (IDR), accuracy (ACC), recall, precision, and F-score. These statistical measures for the proposed method are shown in Table 2. As it can be seen from Table 2, the first 2D-CNN model which receives ACDCT images as input gives an IDR of $85.71 \%$ and an ACC of $84.76 \%$ while the second model fed with MFCC images performs $80.95 \%$ IDR and 79.52\% ACC. After applying mean-value based score fusion, the proposed method achieves an IDR of $90.48 \%$ and an ACC of $88.57 \%$ for 42 people. The confusion matrix for the proposed method is presented in Figure 7. As it can be seen from Figure 7, the proposed method identifies 38 persons out of 42 persons correctly.

Table 2. Classification performance of the proposed method

\begin{tabular}{llllll}
\hline Proposed Method & IDR & ACC & Recall & Precision & F-score \\
\hline ACDCT image & $85.71 \%(36 / 42)$ & $84.76 \%$ & $84.76 \%$ & $81.63 \%$ & $83.17 \%$ \\
CC image & $80.95 \%(34 / 42)$ & $79.52 \%$ & $79.52 \%$ & $75.20 \%$ & $77.30 \%$ \\
Score fusion & $90.48 \%(38 / 42)$ & $88.57 \%$ & $88.57 \%$ & $87.26 \%$ & $87.91 \%$ \\
\hline
\end{tabular}




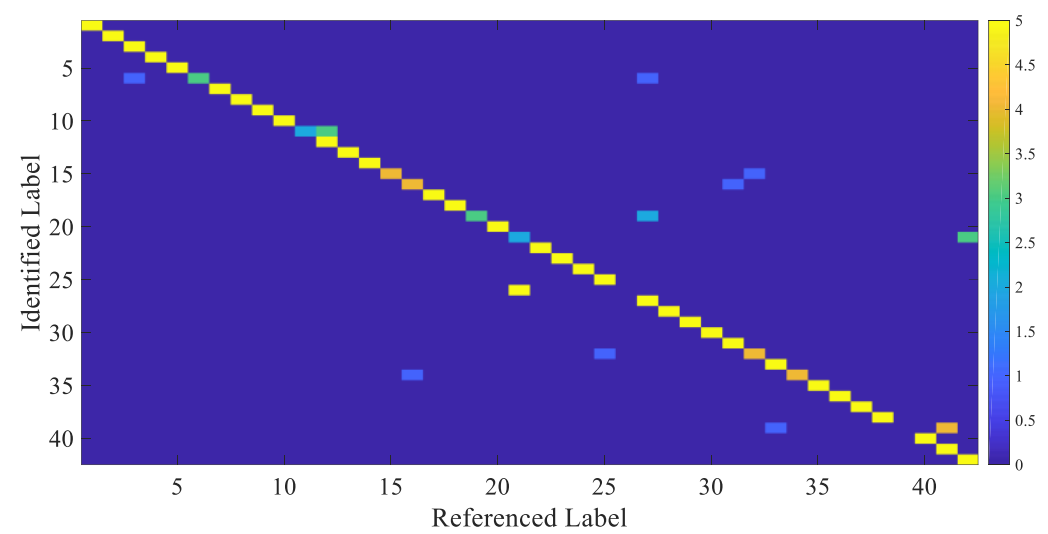

Figure 7. Confusion matrix for the proposed CNN based ECG biometric identification method

The proposed method was compared to both other CNN based methods and conventional methods using ACDCT and cepstral features. For a fair comparison, the methods evaluated with the PTB database were selected. The comparison results according to the IDR and ACC were summarized in Table 3. As seen in Table 3, although the proposed method uses fewer features for more people, it achieves a performance close to that given in [21]. It should be noted that the proposed method has been trained with a small number of data without data augmentation. In addition, the use of a database which consists of non-healthy individuals affected the performance of the proposed method.

Table 3. Comparison results with other methods tested with the PTB database [45]

\begin{tabular}{|c|c|c|c|c|c|}
\hline Author & Method & $\begin{array}{l}\text { Input } \\
\text { Size }\end{array}$ & $\begin{array}{l}\text { \# People } \\
\text { (PTB) }\end{array}$ & Feature Dimensions & Results \\
\hline \multirow{3}{*}{ Gurkan et.al. [21] } & \multirow{3}{*}{ Conventional } & \multirow{3}{*}{-} & \multirow{3}{*}{30} & 1x20 ACDCT & \multirow{3}{*}{ IDR: $97.31 \%$} \\
\hline & & & & $1 \times 13 \mathrm{CC}$ & \\
\hline & & & & 1x200 QRS features & \\
\hline \multirow{2}{*}{ Wang et.al [2] } & \multirow{2}{*}{ Conventional } & \multirow{2}{*}{-} & \multirow{2}{*}{13} & \multirow{2}{*}{$1 \times 20$ ACDCT } & ACC: $94.47 \%$ \\
\hline & & & & & IDR: $100 \%$ \\
\hline Lei et. al. [31] & 1D CNN+SVM & $1 \times 136$ & 100 & $1 \times 160$ & ACC: $99.33 \%$ \\
\hline Labati et.al. [37] & $1 \mathrm{D} \mathrm{CNN}$ & $1 \times 200$ & 52 & - & ACC: $100 \%$ \\
\hline \multirow[t]{2}{*}{ Abdeldayem et.al. [38] } & 2D CNN & $128 \times 128$ & 290 & $1 \times 100$ & IDR: $97.5 \%$ \\
\hline & & & & & ACC: $88.57 \%$ \\
\hline \multirow[t]{2}{*}{ Proposed Method } & 2D CNN & $14 \times 14$ & 42 & $1 \times 128$ & IDR: $90.48 \%$ \\
\hline & & & & & $(38 / 42)$ \\
\hline
\end{tabular}




\section{Conclusion}

A parallel 2D-CNN based ECG biometric identification method has been introduced. The proposed method consists of two 2D-CNN's which operate in parallel and process the ACDCT images and CC images, separately. The experimental results demonstrate that the proposed method yields $88.57 \%$ ACC and 90.48\% IDR. Furthermore, it was observed that the performance of the proposed method is better than that of a 2D-CNN model fed with concatenating ACDCT images with CC images.

\section{Acknowledgments}

This research work was supported by Scientific Research Projects Units, Bursa Technical University, Project Number: $181 \mathrm{~N} 14$.

\section{References}

[1] Jain, A.K., Ross, A. and Prabhakar, S. (2004). An Introduction to Biometric Recognition. IEEE Transactions on Circuits and Systems for Video Technology, 14(1): 4-20.

[2] Wang, Y., Agrafioti, F., Hatzinakos, D. and Plataniotis, K.N. (2008). Analysis of Human Electrocardiogram for Biometric Recognition. EURASIP Journal on Advances in Signal Processing, 2008: 1-11.

[3] Fang, C. and Chan, H.L. (2009). Human Identification by Quantifying Similarity and Dissimilarity in Electrocardiogram Phase Space. Pattern Recognition, 42(9): 1824-1831

[4] Wübbeler, G., Stavridi, M., Kreiseler, D., Bousseljot, R.D. and Elster, C. (2007). Verification of Humans Using Electrocardiogram. Pattern Recognition Letters, 28(10): 1172-1175.

[5] Biel, L., Pettersson, O., Philipson, L. and Wide, P. (2001). ECG Analysis: A New Approach in Human Identification. IEEE Transactions on Instrumentation and Measurement, 50(3): 808-812.

[6] Irvine, J.M., Wiederhold, B.K., Gavshon, L.W., et al. (2001). Heart Rate Variability: A New Biometric for Human Identification. The International Conference on Artificial Intelligence, Las Vegas, Nevada, USA, 25-28 June 2001. pp. 1106-1111.

[7] Shen, T.W., Tompkins, W.J. and Hu, Y.H. (2002). One-lead ECG for Identity Verification. The 2nd Joint Engineering in Medicine and Biology, 24th Annual Conference and the Annual Fall Meeting of the Biomedical Engineering Society, Houston, Texas, USA, 23-26 October 2002. pp. 62-63.

[8] Israel, S.A., Scruggs, W.T., Worek, W.J. and Irvine, J.M. (2003). Fusing Face and ECG for Personal Identification. The 32nd Applied Imagery Pattern Recognition Workshop, Washington, DC, USA, 15-17 October 2003. pp. 226-231.

[9] Israel, S.A., Irvine, J.M., Cheng, A., Wiederhold, M.D. and Wiederhold, B.K. (2005). ECG to Identify Individuals. Pattern Recognition, 38(1): 133-142.

[10] Shen, T.W. (2005). Biometric Identity Verification Based on Electrocardiogram (ECG). Ph.D. Thesis. University of Wisconsin, Madison, USA.

[11] Shen, T.W. (2006). Quartile Discriminant Measurement (QDM) Method for ECG Biometric Feature Selection. International Symposium of Biomedical Engineering, Taiwan, 2006. 
[12] Chuang-Chien, C., Chou-Min, C. and Chih-Yu, H. (2008). A Novel Personal Identity Verification Approach using a Discrete Wavelet Transform of the ECG Signal. International Conference on Multimedia and Ubiquitous Engineering, Busan, Korea, 24-26 April. pp. 201-206.

[13] Irvine, J.M., Israel, S.A., Scruggs, W.T. and Worek, W.J. (2008). EigenPulse: Robust Human Identification from Cardiovascular Function. Pattern Recognition, 41(11): 3427-3435.

[14] Fatemian, S.Z. and Hatzinakos, D. (2009). A New ECG Feature Extractor for Biometric Recognition. The 16th International Conference on Digital Signal Processing, Santorini, Greece, 5-7 July 2009. pp.1-6.

[15] Li, M. and Narayanan, S. (2010). Robust ECG Biometrics by Fusing Temporal and Cepstral Information. 20th IAPR International Conference on Pattern Recognition, İstanbul, Turkey, 23-26 August 2010. pp. 1326-1329.

[16] Sufi, F., Khalil, I. and Habib, I. (2010). Polynomial Distance Measurement for ECG based Biometric Authentication. Security and Communication Networks, 3(4): 303-319.

[17] Loong, J.L.C., Subari, K.S., Besar, R. and Abdullah, M.K. (2010). A New Approach to ECG Biometric Systems: A Comparitive Study between LPC and WPD Systems. World Academy of Science Engineering and Technology, 68: 759-764.

[18] Ting, C.M. and Salleh, S.H. (2010). ECG based Personal Identification using Extended Kalman Filter. 10th Int. Conf. on Information Sciences Signal Processing and Their Applications, Kuala Lumpur, Malaysia, 1013 May 2010. pp. 774-777.

[19] Sufi, F. and Khalil, I. (2011). Faster Person Identification using Compressed ECG in Time Critical Wireless Telecardiology Applications. Journal of Network and Computer Applications, 34(1): 282-293.

[20] Safie, S.I., Soraghan, J.J. and Petropoulakis, L. (2011). Electrocardiogram (ECG) Biometric Authentication using Pulse Active Ratio (PAR). IEEE Transactions on Information Forensics and Security, 6(4): 1315-1322.

[21] Gurkan, H., Guz, U. and Yarman, B.S. (2013). A Novel Biometric Authentication Approach using Electrocardiogram Signals. 35th Annual International IEEE EMBS Conference of the IEEE Engineering in Medicine and Biology Society, Osaka, Japan, 3-7 July 2013. pp. 4259-4262.

[22] Chamatidis, I., Katsika, A. and Spathoulas, G. (2017). Using Deep Learning Neural Networks for ECG based Authentication. International Carnahan Conference on Security Technology, Madrid, Spain, 23-26 October 2017. pp. 1-6.

[23] Chan, A.D.C., Hamdy, M.M., Badre, A. and Badee, V. (2008). Wavelet Distance Measure for Person Identification using Electrocardiograms. IEEE Transactions on Instrumentation and Measurement, 57(2): 248253.

[24] Shen, T.W., Tompkins, W.J. and Hu, Y.H. (2011). Implementation of a One-lead ECG Human Identification System on a Normal Population. Journal of Engineering and Computer Innovations, 2(1): 12-21.

[25] Chen, C.K., Lin, C.L. and Chiu, Y.M. (2011). Individual Identification Based on Chaotic Electrocardiogram Signals. The 6th IEEE Conference on Industrial Electronics and Applications, Beijing, China, 21-23 June 2011. pp. 1765-1770.

[26] Lourenço, A., Silva, H. and Fred, A. (2011). Unveiling the Biometric Potential of Finger-Based ECG Signals. Computational Intelligence and Neuroscience, 2011: 1-8. 
[27] Singh, K., Singhvi, A. and Pathangay, V. (2015). Dry Contact Fingertip ECG based Authentication System using Time, Frequency Domain Features and Support Vector Machine. 37th Annual International Conference of the IEEE. Engineering in Medicine and Biology Society, Milan, Italy, 25-29 August 2015. pp. 526-529.

[28] Wieclaw, L., Khoma, Y., Falat, P., Sabodashko, D. and Herasymenko, V. (2017). Biometric Identification from Raw ECG Signals using Deep Learning Techniques. The 9th International Conference on Intelligent Data Acquisition and Advanced Computing Systems: Technology and Application, Bucharest, Romania, 21-23 September 2017. pp. 129-133.

[29] Guven. G., Gürkan, H. and Guz, U. (2018). Biometric Identification using Fingertip Electrocardiogram Signals. Signal, Image and Video Processing, 12(5): 933-940.

[30] Güven, G. (2016). Fingertip ECG signal based biometric recognition system, Master's Thesis, FMV ISIK University, Istanbul, Turkey.

[31] Şeker, A., Diri, B. And Balık, H.H. (2017). Derin Öğrenme Yöntemleri ve Uygulamaları Hakkında Bir İnceleme. Gazi Mühendislik Bilimleri Dergisi, 3(3): 47-64.

[32] Lei, X., Zhang, Y. and Lu, Z. (2016). Deep Learning Future Representation for Electrocardiogram Identification. IEEE International Conference on Digital Signal Processing, Beijing, China, 16-18 October 2016. pp. 11-14. [26] Lourenço, A., Silva, H. and Fred, A. (2011). Unveiling the Biometric Potential of FingerBased ECG Signals. Computational Intelligence and Neuroscience, 2011: 1-8.

[33] Zhang, Q., Zhou, D. and Zeng, X. (2017). HeartID: A Multiresolution Convolutional Neural Network for ECGbased Biometric Human Identification in Smart Health Applications. IEEE Access, 5: 11805-11816.

[34] Eduardo, A., Aidos, H. and Fred, A. (2017). ECG-based Biometrics using a Deep Autoencoder for Feature Learning: An Empirical Study on Transferability. 6th International Conference on Pattern Recognition Applications and Methods, Porto, Portugal, 24-26 February 2017. pp. 463-470.

[35] Salloum, R. and Kuo, C.C.J. (2017). ECG-based Biometrics using Recurrent Neural Networks. IEEE International Conference on Acoustics Speech and Signal Processing, New Orleans, LA, USA, 5-9 March 2017. pp. 2062-2066

[36] Zhang, Q., Zhou, D. and Zeng, X. (2017). PulsePrint: Single-arm-ECG Biometric Human Identification using Deep Learning. 8th IEEE 8th Annual Ubiquitous Computing Electronics and Mobile Communication Conference, New York, NY, USA, 19-21 October 2017. pp. 452-456.

[37] Luz, E.J.S., Moreira, G.J.P., Oliveira, L.S., Schwartz, W.R. and Menotti, D. (2018). Learning Deep off-theperson Heart Biometrics Representations. IEEE Transactions on Information Forensics and Security, 13(5): 1258-1270.

[38] Labati, R.D., Muñoz, E., Piuri, V., Sassi, R. and Scotti, F. (2018). Deep-ECG:Convolutional Neural Networks for ECG Biometric Recognition. Pattern Recognition Letters, doi: 10.1016/j.patrec.2018.03.028 (Article in Press).

[39] Abdeldayem, S.S. and Bourlai, T. (2018). ECG-based Human Authentication using High-level Spectrotemporal Signal Features. IEEE International Conference on Big Data, Seattle, WA, USA, 10-13 December 2018. pp. 4984-4993.

[40] Goldberger, A.L., Amaral, L.A.N., Glass, L., et al. (2000). PhysioBank, PhysioToolkit, and PhysioNet: Components of a New Research Resource for Complex Physiologic Signals. Circulation, 101(23): e215-e220. 
[41] Bousseljot, R., Kreiseler, D. and Schnabel, A. (1995). Nutzung der EKGSignaldatenbank CARDIODAT der PTB über das Internet. Biomedizinische Technik, 40(1): 317.

[42] Li, M., Rozgic, V., Thatte, G., Lee, S., Emken, A., Annavaram, M., Mitra, U., Sprujit-Metz, D. and Narayanan, S. (2010). Multimodal Physical Activity Recognition by Fusing Temporal and Cepstral Information. IEEE Transactions on Neural Systems and Rehabilitation Engineering, 18 (4): 369-380.

[43] Huang, X., Acero, A. and Hon, H.W. (2001). Spoken Language Processing: A Guide to Theory, Algorithm, and System Development. Prentice-Hall, Upper Saddle River, New Jersey, 1st Edition, 980, ISBN: 0-13022616-5.

[44] Young, S., Evermann, G., Gales, M., Hain, T., Kershaw, D., Liu, X., et al. (2006). The HTK Book (for HTK Version 3.4.1), Cambridge University Engineering Department.

[45] Pinto, C.R., Cardoso, J.S. and Lourenço, A. (2018). Evolution, Current Challanges and Future Possibilities in ECG Biometrics. IEEE Access, 6: 34746-34776. 